\title{
On convergence of branched continued fraction expansions of Horn's hypergeometric function $H_{3}$ ratios
}

\begin{abstract}
Antonova T.M.
The paper deals with the problem of convergence of the branched continued fractions with two branches of branching which are used to approximate the ratios of Horn's hypergeometric function $H_{3}(a, b ; c ; \mathbf{z})$. The case of real parameters $c \geq a \geq 0, c \geq b \geq 0, c \neq 0$, and complex variable $\mathbf{z}=\left(z_{1}, z_{2}\right)$ is considered. First, it is proved the convergence of the branched continued fraction for $\mathbf{z} \in G_{\mathbf{h}}$, where $G_{\mathbf{h}}$ is two-dimensional disk. Using this result, sufficient conditions for the uniform convergence of the above mentioned branched continued fraction on every compact subset of the domain $H=\bigcup_{\varphi \in(-\pi / 2, \pi / 2)} G_{\varphi}$, where

$$
\begin{aligned}
G_{\varphi}=\left\{\mathbf{z} \in \mathbb{C}^{2}:\right. & \operatorname{Re}\left(z_{1} e^{-i \varphi}\right)<\lambda_{1} \cos \varphi,\left|\operatorname{Re}\left(z_{2} e^{-i \varphi}\right)\right|<\lambda_{2} \cos \varphi \\
& \left.\left|z_{k}\right|+\operatorname{Re}\left(z_{k} e^{-2 i \varphi}\right)<v_{k} \cos ^{2} \varphi, k=1,2 ;\left|z_{1} z_{2}\right|-\operatorname{Re}\left(z_{1} z_{2} e^{-2 \varphi}\right)<v_{3} \cos ^{2} \varphi\right\}
\end{aligned}
$$
\end{abstract}

are established. gence.

Key words and phrases: Horn's hypergeometric function $\mathrm{H}_{3}$, branched continued fraction, conver-

Lviv Polytechnic National University, 12 Bandera str., 79013, Lviv, Ukraine

E-mail: tamara.m.antonova@lpnu.ua

\section{Introduction}

Solving various problems of applied mathematics involves the use of special functions. An important class of special functions is hypergeometric functions, among which the hypergeometric function of Gauss should be noted at the first place. This function is a solution to the ordinary differential equation [13, p. 56]. Usually, the literature provides a definition of the hypergeometric function of Gauss with the help of a hypergeometric series. In 1889, J. Horn gave the definition of a hypergeometric series of two variables. He studied, in particular, the series of the second order. The list of the series, regions of convergence and corresponding systems of differential equations in partial derivatives are given in [13, pp. 224-229].

It is known that continued fractions have numerous applications in the theory of approximation of functions of one variable $[15,16]$. Multidimensional generalizations of continued fractions can be considered as a tool of rational approximation of functions of several variables $[11,12]$. In particular, branched continued fractions $(B C F)$ of the form

$$
d_{0}(\mathbf{z})+D_{k=1}^{\infty} \sum_{i_{k}=1}^{2} \frac{c_{i(k)}(\mathbf{z})}{d_{i(k)}(\mathbf{z})}
$$

where $i(k)=\left(i_{1}, i_{2}, \ldots, i_{k}\right)$ is a multiindex, $\mathcal{I}=\left\{i(k): i_{r}=1,2 ; 1 \leq r \leq k, k \geq 1\right\}$ is a set of 
multiindices, the $d_{0}(\mathbf{z})$ and the elements $c_{i(k)}(\mathbf{z})$ and $d_{i(k)}(\mathbf{z}), i(k) \in \mathcal{I}$, are certain polynomials, that usually used to approximate the ratios of some hypergeometric functions from the Horn's list $[14,17]$. In this case, we need to solve the following problems:

- to construct the expansion of ratio of multiple hypergeometric functions into BCF;

- to investigate the convergence of this expansion;

- to prove that the BCF converges to a function which is an analytic continuation of the ratio of multiple hypergeometric function in some domain.

Some approaches for solving these problems for ratios of the Horn hypergeometric function $\mathrm{H}_{3}$ (see [13]), which is defined by double power series

$$
H_{3}(a, b ; c ; \mathbf{z})=\sum_{m, n=0}^{\infty} \frac{(a)_{2 m+n}(b)_{n}}{(c)_{m+n}} \frac{z_{1}^{m}}{m !} \frac{z_{2}^{n}}{n !}, \quad\left|z_{1}\right|<r, \quad\left|z_{2}\right|<s, \quad r+\left(s-\frac{1}{2}\right)^{2}=\frac{1}{4}
$$

are described in [5]. In the series (2), the parameters $a, b$ and $c$ are complex constants, $c$ is not a non-positive integer, $\mathbf{z}=\left(z_{1}, z_{2}\right) \in \mathbb{C}^{2},(\cdot)_{k}$ is the Pochhammer symbol defined for any complex number $\alpha$ and non-negative integer $k$ by $(\alpha)_{0}=1$ and $(\alpha)_{k}=\alpha(\alpha+1) \ldots(\alpha+k-1)$.

In the above mentioned paper, the ratios

$$
R_{k}(a, b ; c ; \mathbf{z})=\frac{H_{3}(a, b ; c ; \mathbf{z})}{H_{3}\left(a+\delta_{k}^{1}, b+\delta_{k}^{2} ; c+1 ; \mathbf{z}\right)}, \quad k=1,2,
$$

where $\delta_{k}^{j}$ is the Kronecker delta, are considered.

Using the recurrence relations for the function (2), the formal expansions for

$$
\left(1-4 z_{1} \delta_{i_{0}}^{2}\right) R_{i_{0}}(a, b ; c ; \mathbf{z}), \quad i_{0}=1,2,
$$

into BCF of the form (1) are constructed. The elements of these BCF are defined as follows

$$
\begin{gathered}
d_{0}(\mathbf{z})=1+\left(\frac{b+1-a}{c} z_{2}-2 \frac{2 c-a}{c} z_{1}\right) \delta_{i_{0^{\prime}}}^{2} \\
c_{1}(\mathbf{z})=-\frac{\left(2 c+\delta_{i_{0}}^{2}-a-2 \delta_{i_{0}}^{2}(2 c-a-b) z_{2}\right)\left(a+\delta_{i_{0}}^{1}\right) z_{1}}{c(c+1)} \\
c_{2}(\mathbf{z})=-\frac{\left(b+\delta_{i_{0}}^{2}\right)\left(c+\delta_{i_{0}}^{2}-a\right)\left(1-4 z_{1}\right) z_{2}}{c(c+1)},
\end{gathered}
$$

and

$$
\begin{gathered}
d_{i(k)}(\mathbf{z})=1+\frac{b+\sum_{p=0}^{k-1}\left(\delta_{i_{p}}^{2}-\delta_{i_{p}}^{1}\right)+1-a}{c+k} \delta_{i_{k}}^{2} z_{2}-2 \frac{2 c-a+k+\sum_{p=0}^{k-1} \delta_{i_{p}}^{2}}{c+k} \delta_{i_{k}}^{2} z_{1}, \\
c_{i(k), 1}(\mathbf{z})=-\frac{\left(2 c-a+k+\sum_{p=0}^{k-1} \delta_{i_{p}}^{2}-2 \delta_{i_{k}}^{2}(2 c-a-b+k) z_{2}\right)\left(a+\sum_{p=0}^{k-1} \delta_{i_{p}}^{1}\right) z_{1}}{(c+k)(c+k+1)}, \\
c_{i(k), 2}(\mathbf{z})=-\frac{\left(b+\sum_{p=0}^{k-1} \delta_{i_{p}}^{2}\right)\left(c-a+\sum_{p=0}^{k-1} \delta_{i_{p}}^{2}\right)\left(1-4 z_{1}\right) z_{2}}{(c+k)(c+k+1)}
\end{gathered}
$$

for all $i(k) \in \mathcal{I}$. 
In this paper we continue to investigate the convergence of the obtained BCF. Let us recall some basic concepts and notations. Finite BCF

$$
f_{n}(\mathbf{z})=d_{0}(\mathbf{z})+D_{k=1}^{n} \sum_{i_{k}=1}^{2} \frac{c_{i(k)}(\mathbf{z})}{d_{i(k)}(\mathbf{z})}
$$

is called the $n$th approximant of the BCF (1). Note that for each $n \in \mathbb{N}$ the approximant $f_{n}(\mathbf{z})$ can also be written as

$$
f_{n}(\mathbf{z})=d_{0}(\mathbf{z})+\sum_{i_{1}=1}^{2} \frac{c_{i(1)}(\mathbf{z})}{Q_{i(1)}^{(n)}(\mathbf{z})},
$$

where the tails $Q_{i(k)}^{(n)}(\mathbf{z}), i(k) \in \mathcal{I}, 1 \leq k \leq n$, are defined as follows

$$
\begin{gathered}
Q_{i(n)}^{(n)}(\mathbf{z})=d_{i(n)}(\mathbf{z}), \quad n \geq 1, \\
Q_{i(k)}^{(n)}(\mathbf{z})=d_{i(k)}(\mathbf{z})+\sum_{i_{k+1}=1}^{2} \frac{c_{i(k+1)}(\mathbf{z})}{Q_{i(k+1)}^{(n)}(\mathbf{z})}, \quad i(k) \in \mathcal{I}, \quad 1 \leq k \leq n-1, \quad n \geq 2 .
\end{gathered}
$$

The BCF (1) and the BCF

$$
d_{0}^{*}(\mathbf{z})+D_{k=1}^{\infty} \sum_{i_{k}=1}^{2} \frac{c_{i(k)}^{*}(\mathbf{z})}{d_{i(k)}^{*}(\mathbf{z})},
$$

with $n$th approximants $f_{n}$ and $f_{n}^{*}$, respectively, are said to be equivalent if $f_{n}=f_{n}^{*}, n \geq 1$. The $\mathrm{BCF}$

$$
d_{0}(\mathbf{z})+D_{k=1}^{\infty} \sum_{i_{k}=1}^{2} \frac{c_{i(k)}(\mathbf{z}) \rho_{i(k-1)} \rho_{i(k)}}{d_{i(k)}(\mathbf{z}) \rho_{i(k)}},
$$

where $\rho_{i(0)}=1, \rho_{i(k)}, i(k) \in \mathcal{I}, k \geq 1$, are non-zero complex numbers, is equivalent to the BCF (1).

The BCF (1), whose elements are functions of two variables in the certain domain $D$, $D \subset \mathbb{C}^{2}$, is called uniformly convergent on set $E, E \subset D$, if sequence of its approximants $\left\{f_{n}(\mathbf{z})\right\}$ converges uniformly on $E$. When this occurs for an arbitrary set $E$ such that $\bar{E} \subset D$ (here $\bar{E}$ is the closure of the set $E$ ) we say that the BCF converges uniformly on every compact subset of $D$.

Note that some interesting and effective methods for studying the convergence of branched continued fractions are considered in works $[1,3,4,7,8,10]$.

In what follows, we will use the following auxiliary statements.

Proposition 1. Let elements of the BCF (1) be the functions defined in some domain $D$, $D \subset \mathbb{C}^{2}$. If there exist positive functions $g_{i(k)}(\mathbf{z})$ given in the domain $D$ such that for each $\mathbf{z} \in D$ and for all possible values of multiindices $i(k) \in \mathcal{I}$ the following condition

$$
\left|d_{i(k)}(\mathbf{z})\right| \geq g_{i(k)}(\mathbf{z})+\sum_{i_{k+1}=1}^{2} \frac{\left|c_{i(k+1)}(\mathbf{z})\right|}{g_{i(k+1)}(\mathbf{z})}
$$

holds, then BCF (1) converges for each $\mathbf{z} \in D$ and

$$
\left|f_{n}(\mathbf{z})-d_{0}(\mathbf{z})\right| \leq \sum_{i_{1}=1}^{2} \frac{\left|c_{i(1)}(\mathbf{z})\right|}{g_{i(1)}(\mathbf{z})} .
$$


The correctness of this statement can be verified by the scheme of proving the generalization of the Sleshinsky-Pringsheim criterion for other multidimensional generalizations of continued fractions [2].

Proposition 2. Let elements of the BCF (1) be the functions defined in some domain $D$, $D \subset \mathbb{C}^{2}$, and the following conditions for each $\mathbf{z} \in D$ and for all possible values of multiindices $i(k) \in \mathcal{I}$ are valid:

(1) $\operatorname{Re} d_{i(k)}(\mathbf{z})>0$;

(2) there exist functions $g_{i(k)}(\mathbf{z})$ given in the domain $D$ such that $0<g_{i(k)}(\mathbf{z}) \leq \operatorname{Re} d_{i(k)}(\mathbf{z})$ and

$$
\sum_{i_{k+1}=1}^{2} \frac{\left|c_{i(k+1)}(\mathbf{z})\right|-\operatorname{Re} c_{i(k+1)}(\mathbf{z})}{g_{i(k+1)}(\mathbf{z})} \leq 2\left(\operatorname{Re} d_{i(k)}(\mathbf{z})-g_{i(k)}(\mathbf{z})\right) .
$$

Then for each $n \geq 1$

$$
\operatorname{Re}\left(Q_{i(k)}^{(n)}(\mathbf{z})\right) \geq g_{i(k)}(\mathbf{z}) \quad \text { for all } \quad i(k) \in \mathcal{I}, \quad 1 \leq k \leq n, \quad \text { and } \quad \mathbf{z} \in D,
$$

where $Q_{i(k)}^{(n)}(\mathbf{z}), i(k) \in \mathcal{I}, 1 \leq k \leq n, n \geq 1$, defined by (9) and (10).

This statement follows from [6, Lemma 1] for $N=2$.

Theorem 1. Let $\left\{f_{n}(\mathbf{z})\right\}$ be a sequence of functions, holomorphic in the domain $D, D \subset \mathbb{C}^{2}$, which is uniformly bounded on every compact subset of $D$. Let this sequence converges at each point of the set $E, E \subset D$, which is the 4-dimensional neighborhood of the point $\mathbf{z}^{0}$, $\mathbf{z}^{\mathbf{0}} \in D$. Then $\left\{f_{n}(\mathbf{z})\right\}$ converges uniformly on every compact subset of the domain $D$ to a function holomorphic in $D$.

This theorem follows from [9, Theorem 2.17 ] for $N=2$.

\section{Main results}

Using the method of studying the convergence of branched continued fractions, which was described in the article [5], some new sufficient conditions for convergence of BCF are established.

Theorem 2. Let parameters of hypergeometric function $H_{3}(a, b ; c ; \mathbf{z})$ are such that

$$
c \geq a \geq 0, \quad c \geq b \geq 0 .
$$

Then the BCF (1) with $d_{0}(\mathbf{z}), c_{i(k)}(\mathbf{z})$, and $d_{i(k)}(\mathbf{z}), i(k) \in \mathcal{I}$, defined by (3)-(8), for $i_{0}=1$ converges to a finite value $f(\mathbf{z})$ for each $\mathbf{z} \in G_{\mathbf{h}}$, where

$$
G_{\mathbf{h}}=\left\{\mathbf{z} \in \mathbb{C}^{2}:\left|z_{1}\right| \leq h_{1},\left|z_{2}\right| \leq h_{2}\right\},
$$

and $h_{1}, h_{2}$ are positive numbers such that

$$
8 h_{1}\left(1+2 h_{2}\right)\left(1-4 h_{1}-h_{2}\right)+4\left(1+4 h_{1}\right) h_{2} \leq\left(1-4 h_{1}-h_{2}\right)^{2} ;
$$

it converges uniformly on every compact subset of $\operatorname{Int} G_{\mathbf{h}}$ to a function $f(\mathbf{z})$ holomorphic in Int $G_{\mathbf{h}}$. 
Proof. Let $i(k) \in \mathcal{I}$ and $\mathbf{z} \in G_{\mathbf{h}}$ with positive numbers $h_{1}$ and $h_{2}$. If $2 g_{i(k)}(\mathbf{z})=\left|d_{i(k)}(\mathbf{z})\right|$, then condition (12) takes the form

$$
\sum_{i_{k+1}=1}^{2} \frac{\left|c_{i(k+1)}(\mathbf{z})\right|}{\left|d_{i(k)}(\mathbf{z}) d_{i(k+1)}(\mathbf{z})\right|} \leq \frac{1}{4}
$$

Let us estimate elements of the investigated fractions under conditions (13)-(15) and $i_{0}=1$. If $i_{k}=1$, then $d_{i(k)}(\mathbf{z})=1$. If $i_{k}=2$, then

$$
\begin{aligned}
\left|d_{i(k)}(\mathbf{z})\right| & \geq 1-\frac{\left|b+\sum_{p=0}^{k-1}\left(\delta_{i_{p}}^{2}-\delta_{i_{p}}^{1}\right)+1-a\right|}{c+k}\left|z_{2}\right|-4\left|z_{1}\right| \frac{2 c-a+2 k-1}{2 c+2 k} \\
& \geq 1-\frac{|b-a|+k-1}{c+k}\left|z_{2}\right|-4\left|z_{1}\right| \frac{2 c-a+2 k-1}{2 c+2 k}>1-\left|z_{2}\right|-4\left|z_{1}\right| \geq 1-h_{2}-4 h_{1} .
\end{aligned}
$$

Also, then

$$
\begin{gathered}
\left|c_{1}(\mathbf{z})\right|=\frac{(2 c-a)(a+1)}{c(c+1)}\left|z_{1}\right| \leq 2\left|z_{1}\right| \leq 2\left|h_{1}\right|, \\
\left|c_{2}(\mathbf{z})\right|=\frac{b(c-a)\left|\left(1-4 z_{1}\right) z_{2}\right|}{c(c+1)} \leq\left(1+4\left|z_{1}\right|\right)\left|z_{2}\right| \leq\left(1+4 h_{1}\right) h_{2},
\end{gathered}
$$

and

$$
\begin{aligned}
\left|c_{i(k), 1}(\mathbf{z})\right| & =\frac{\left|2 c-a+k+\sum_{p=0}^{k} \delta_{i_{p}}^{2}-2 \delta_{i_{k}}^{2}(2 c-a-b+k) z_{2}\right|\left(a+\sum_{p=0}^{k} \delta_{i_{p}}^{1}\right)}{(c+k)(c+k+1)}\left|z_{1}\right| \\
& \leq \frac{\left(2 c-a+2 k+2 \delta_{i_{k}}^{2}(2 c-a-b+k)\left|z_{2}\right|\right)(a+k+1)}{(c+k)(c+k+1)}\left|z_{1}\right| \\
& \leq \frac{(2 c-a+2 k)\left(1+2\left|z_{2}\right|\right)(a+k+1)}{(c+k)(c+k+1)}\left|z_{1}\right| \leq 2\left(1+2\left|z_{2}\right|\right)\left|z_{1}\right| \leq 2 h_{1}\left(1+2 h_{2}\right), \\
\left|c_{i(k), 2}(\mathbf{z})\right| & \leq \frac{\left(c-a+\sum_{p=0}^{k} \delta_{i_{p}}^{2}\right)\left(b+\sum_{p=0}^{k} \delta_{i_{p}}^{2}\right)}{(c+k)(c+k+1)}\left(1+4\left|z_{1}\right|\right)\left|z_{2}\right| \\
& \leq \frac{(c-a+k)(b+k)}{(c+k)(c+k+1)}\left(1+4\left|z_{1}\right|\right)\left|z_{2}\right| \leq\left(1+4\left|z_{1}\right|\right)\left|z_{2}\right| \leq\left(1+4 h_{1}\right) h_{2} .
\end{aligned}
$$

Hence it follows that

$$
\sum_{i_{k+1}=1}^{2} \frac{\left|c_{i(k+1)}(\mathbf{z})\right|}{\left|d_{i(k)}(\mathbf{z})\right|\left|d_{i(k+1)}(\mathbf{z})\right|} \leq \frac{2 h_{1}\left(1+2 h_{2}\right)}{1-4 h_{1}-h_{2}}+\frac{h_{2}\left(1+4 h_{1}\right)}{\left(1-4 h_{1}-h_{2}\right)^{2}} \leq \frac{1}{4}
$$

therefore, according to Proposition 1 and Theorem 1, the BCF converges for each $\mathbf{z} \in G_{\mathbf{h}}$ to a function $f(\mathbf{z})$. Convergence is uniform on every compact subset of $\operatorname{Int} G_{\mathbf{h}}$, and $f(\mathbf{z})$ is holomorphic in Int $G_{\mathbf{h}}$.

Remark 1. It is easy to check that in the case of $h_{1}=h_{2}=1 / 25$ the inequality (15) is valid. 
Theorem 3. Let the parameters $a, b$, and $c$ of the hypergeometric function (2) satisfy inequalities (13), $\lambda_{1}, \lambda_{2}, \mu_{1}, \mu_{2}, v_{1}, v_{2}$, and $v_{3}$ be positive numbers such that

$$
\frac{v_{2}+4 v_{3}}{\mu_{2}} \leq \min \left(2\left(1-\mu_{1}\right)-\frac{2 v_{1}}{\mu_{1}}, 2\left(1-4 \lambda_{1}-\lambda_{2}-\mu_{2}\right)-\frac{2 v_{1}+4 v_{3}}{\mu_{1}}\right) .
$$

Then the BCF (1), where $d_{0}(\mathbf{z}), c_{i(k)}(\mathbf{z})$ and $d_{i(k)}(\mathbf{z}), i(k) \in \mathcal{I}$, defined by (3)-(8), for $i_{0}=1$, converges uniformly on every compact subset of $H=\bigcup_{\varphi \in(-\pi / 2, \pi / 2)} G_{\varphi}$, where

$$
\begin{aligned}
G_{\varphi}=\{\mathbf{z} \in & \mathbb{C}^{2}: \operatorname{Re}\left(z_{1} e^{-i \varphi}\right)<\lambda_{1} \cos \varphi,\left|\operatorname{Re}\left(z_{2} e^{-i \varphi}\right)\right|<\lambda_{2} \cos \varphi, \\
& \left.\left|z_{k}\right|+\operatorname{Re}\left(z_{k} e^{-2 i \varphi}\right)<v_{k} \cos ^{2} \varphi, k=1,2 ;\left|z_{1} z_{2}\right|-\operatorname{Re}\left(z_{1} z_{2} e^{-2 \varphi}\right)<v_{3} \cos ^{2} \varphi\right\},
\end{aligned}
$$

to a function $f(\mathbf{z})$ holomorphic in $H$.

Proof. Let for all $i(k) \in \mathcal{I}$

$$
\rho_{i(k)}=e^{-i \varphi}, \quad \varphi \in(-\pi / 2, \pi / 2) .
$$

We will estimate the elements of the BCF of the form (11), equivalent to the investigated BCF, under conditions (13), (16) and $i_{0}=1$ in the domain (17).

Let $i(k) \in \mathcal{I}, \varphi$ be an arbitrary real in $(-\pi / 2, \pi / 2)$ and $\mathbf{z}$ be an arbitrary point in $G_{\varphi}$. If $i_{k}=1$, then

$$
\operatorname{Re}\left(d_{i(k)}(\mathbf{z}) e^{-i \varphi}\right)=\cos \varphi>\mu_{1} \cos \varphi
$$

If $i_{k}=2$, then

$$
\begin{gathered}
\operatorname{Re}\left(d_{i(k)}(\mathbf{z}) e^{-i \varphi}\right)=\cos \varphi+\frac{b+\sum_{p=0}^{k-1}\left(\delta_{i_{p}}^{2}-\delta_{i_{p}}^{1}\right)+1-a}{c+k} \operatorname{Re}\left(z_{2} e^{-i \varphi}\right) \\
-2 \frac{2 c-a+k+\sum_{p=0}^{k-1} \delta_{i_{p}}^{2}}{c+k} \operatorname{Re}\left(z_{1} e^{-i \varphi}\right) .
\end{gathered}
$$

Taking into account condition (13), we obtain

$$
-c-k \leq-a-k<b+\sum_{p=0}^{k-1}\left(\delta_{i_{p}}^{2}-\delta_{i_{p}}^{1}\right)+1-a<b+k \leq c+k
$$

and

$$
0<2 c-a+k+\sum_{p=0}^{k-1} \delta_{i_{p}}^{2}<2 c+2 k-a-1<2 c+2 k
$$

hence

$$
\operatorname{Re}\left(d_{i(k)}(\mathbf{z}) e^{-i \varphi}\right)>\left(1-4 \lambda_{1}-\lambda_{2}\right) \cos \varphi>\mu_{2} \cos \varphi .
$$

Further,

$$
\begin{aligned}
\left|c_{i(k), 1}(\mathbf{z})\right|-\operatorname{Re}\left(c_{i(k), 1}\left(\mathbf{z} e^{-2 i \varphi}\right) \leq\right. & \frac{\left(a+\sum_{p=0}^{k} \delta_{i_{p}}^{1}\right)\left(2 c-a+k+\sum_{p=0}^{k} \delta_{i_{p}}^{2}\right)}{(c+k)(c+k+1)}\left(\left|z_{1}\right|+\operatorname{Re}\left(z_{1} e^{-2 i \varphi}\right)\right) \\
& +\frac{2 \delta_{i_{k}}^{2}\left(a+\sum_{p=0}^{k} \delta_{i_{p}}^{1}\right)(2 c-a-b+k)}{(c+k)(c+k+1)}\left(\left|z_{1} z_{2}\right|-\operatorname{Re}\left(z_{1} z_{2}\right)\right) \\
< & 2\left(\left|z_{1}\right|+\operatorname{Re}\left(z_{1} e^{-2 i \varphi}\right)\right)+4 \delta_{i_{k}}^{2}\left(\left|z_{1} z_{2}\right|-\operatorname{Re}\left(z_{1} z_{2}\right)\right) \\
\leq & \left(2 v_{1}+4 \delta_{i_{k}}^{2} v_{3}\right) \cos ^{2} \varphi
\end{aligned}
$$


and

$$
\begin{aligned}
\left|c_{i(k), 2}(\mathbf{z})\right|-\operatorname{Re}\left(c_{i(k), 2} \mathbf{z} e^{-2 i \varphi}\right)< & \frac{\left(c-a+\sum_{p=0}^{k} \delta_{i_{p}}^{2}\right)\left(b+\sum_{p=0}^{k} \delta_{i_{p}}^{2}\right)}{(c+k)(c+k+1)}\left(\left|z_{2}\right|+\operatorname{Re}\left(z_{2} e^{-2 i \varphi}\right)\right. \\
& \left.+4\left|z_{1} z_{2}\right|-4 \operatorname{Re}\left(z_{1} z_{2} e^{-2 i \varphi}\right)\right) \leq\left(v_{2}+4 v_{3}\right) \cos ^{2} \varphi
\end{aligned}
$$

hence, for all $i(k) \in \mathcal{I}$,

$$
\begin{aligned}
\sum_{i_{k+1}=1}^{2} \frac{\left|c_{i(k+1)}(\mathbf{z})\right|-\operatorname{Re} c_{i(k+1)}\left(\mathbf{z} e^{-2 i \varphi}\right)}{\mu_{i_{k+1}} \cos \varphi} & \leq\left(\frac{2 v_{1}}{\mu_{1}}+\frac{v_{2}+4 v_{3}}{\mu_{2}}\right) \cos \varphi \leq 2\left(1-\mu_{1}\right) \cos \varphi \\
& =2\left(\operatorname{Re}\left(d_{i(k)} e^{-i \varphi}\right)-\mu_{1} \cos \varphi\right), \quad i_{k}=1,
\end{aligned}
$$

and

$$
\begin{aligned}
\sum_{i_{k+1}=1}^{2} \frac{\left|c_{i(k+1)}(\mathbf{z})\right|-\operatorname{Re} c_{i(k+1)}\left(\mathbf{z} e^{-2 i \varphi}\right)}{\mu_{i_{k+1}} \cos \varphi} & \leq\left(\frac{2 \nu_{1}+4 v_{3}}{\mu_{1}}+\frac{\nu_{2}+4 v_{3}}{\mu_{2}}\right) \cos \varphi \\
& \leq 2\left(1-4 \lambda_{1}-\lambda_{2}-\mu_{2}\right) \cos \varphi \\
& <2\left(\operatorname{Re}\left(d_{i(k)} e^{-i \varphi}\right)-\mu_{2} \cos \varphi\right), \quad i_{k}=2 .
\end{aligned}
$$

It follows from (19), (20) that for the elements of the BCF (11), where $\rho_{i(k)}$ is defined by (18), the conditions of Proposition 2, where $g_{i(k)}(\mathbf{z})=\mu_{i_{k}}, i(k) \in \mathcal{I}$, are satisfied. Therefore, for the tails of the BCF (1), whose elements are defined by (3)-(8), the inequalities

$$
\operatorname{Re}\left(Q_{i(k)}^{(n)}(\mathbf{z}) e^{-i \varphi}\right) \geq \mu_{i_{k}} \cos \varphi, \quad i(k) \in \mathcal{I},
$$

are valid. This means that the approximants of investigated BCF are holomorphic functions in the domain $G_{\varphi}$ and, consequently, by virtue of arbitrariness of $\varphi$, for all $\mathbf{z} \in H$.

Let $K_{\varphi}$ be an arbitrary compact subset of the domain $G_{\varphi}$. Then there exists an open ball $B_{\varphi}$ with center in the origin and radius $r_{\varphi}$ such that $K_{\varphi} \subset B_{\varphi}$. Taking into account estimate (21), we obtain for the arbitrary $\mathbf{z} \in K_{\varphi}$ and any $n \geq 1$

$$
\begin{aligned}
\left|f_{n}(\mathbf{z})\right| \leq 1+\sum_{i_{1}=1}^{2} \frac{\left|c_{i(1)}(\mathbf{z})\right|}{\left|Q_{i(1)}^{(n)}(\mathbf{z})\right|} \leq 1 & +\frac{(2 c-a)(a+1)}{\mu_{1} c(c+1) \cos \varphi} r_{\varphi} \\
& +\frac{b(c-a)}{\mu_{2} c(c+1) \cos \varphi}\left(1+4 r_{\varphi}\right) r_{\varphi}=M\left(K_{\varphi}\right),
\end{aligned}
$$

i.e. $\left\{f_{n}(\mathbf{z})\right\}$ is a uniformly bounded sequence on $K_{\varphi}$. Thus, this sequence is uniformly bounded on every compact subset of the domain (17).

Let $K$ be an arbitrary compact subset of $H$. Let us cover $K$ with domains of the form $G_{\varphi}$. From this cover we choose the finite subcover

$$
G_{\varphi^{(1)}}, G_{\varphi^{(2)}}, \ldots, G_{\varphi^{(k)}} .
$$

Set $M(K)=\max _{1 \leq r \leq k} M\left(G_{\varphi^{(r)}}\right)$. Then for arbitrary $\mathbf{z} \in K$ we obtain $|f(\mathbf{z})| \leq M(K)$, for $n \geq 1$, i.e. the sequence $f(\mathbf{z})$ is uniformly bounded on every compact subset of $H$.

Further, we set

$$
\gamma=\min \left(\lambda_{1} \cos \varphi, \lambda_{2} \cos \varphi, \frac{\nu_{1}}{2} \cos ^{2} \varphi, \frac{\nu_{2}}{2} \cos ^{2} \varphi, \sqrt{\frac{\nu_{3}}{2}} \cos \varphi, \frac{1}{25}\right) .
$$


Let $G_{\gamma}=\left\{\mathbf{z} \in \mathbb{C}^{2}:\left|z_{1}\right|<\gamma,\left|z_{2}\right|<\gamma\right\}$. If $\mathbf{z} \in G_{\gamma}$, then elements of the BCF (1) satisfy the conditions of Theorem 2, and this BCF converges. Since $G_{\gamma} \subset G_{\varphi} \subset H$ and $G_{\gamma}$ is the neighborhood of the point $\mathbf{z}=\mathbf{0}$, then, according to Theorem 1 , the BCF converges uniformly on compact subsets of $H$ to a function $f(\mathbf{z})$ holomorphic in $H$.

Corollary 1. Let $\mu_{1}, \mu_{2}, v_{2}, \lambda_{2}$ be positive numbers such that

$$
\frac{v_{2}}{\mu_{2}}<\min \left(2\left(1-\mu_{1}\right), 2\left(1-\lambda_{2}-\mu_{2}\right)\right) .
$$

Then the BCF (1), whose elements are defined by (3)-(8), where $i_{0}=1$, converges at each point $\mathbf{z} \in P_{\varphi}$, where

$$
P_{\varphi}=\left\{\mathbf{z} \in \mathbb{C}^{2}: z_{1}=\left|z_{1}\right| e^{i(\pi+2 \varphi)}, z_{2}=\left|z_{2}\right| e^{i \pi},\left|z_{2}\right|<\min \left(\lambda_{2}, v_{2} / 2\right)\right\}, \quad|\varphi| \leq \pi / 4 .
$$

Proof. Indeed, if condition (22) is satisfied, then there are positive constants $\lambda_{1}, v_{1}, v_{3}$, such that conditions (16) are satisfied. Let us show that $P_{\varphi} \subset G_{\varphi}$. For any $\varphi \in[-\pi / 4, \pi / 4]$ and $\mathbf{z} \in P_{\varphi}$ we have

$$
\begin{gathered}
\left|z_{1}\right|+\operatorname{Re}\left(z_{1} e^{-2 i \varphi}\right)=\left|z_{1}\right|+\operatorname{Re}\left(\left|z_{1}\right| e^{i \pi}\right)=0, \\
\left|z_{2}\right|+\operatorname{Re}\left(z_{2} e^{-2 i \varphi}\right)=\left|z_{2}\right|+\operatorname{Re}\left(\left|z_{2}\right| e^{i(\pi-2 \varphi)}\right)=\left|z_{2}\right|(1-\cos (2 \varphi))<v_{2} \cos ^{2} \varphi, \\
\left|z_{1} z_{2}\right|-\operatorname{Re}\left(z_{1} z_{2} e^{-2 i \varphi}\right)=\left|z_{1} z_{2}\right|-\operatorname{Re}\left(\left|z_{1} z_{2}\right| e^{2 i \pi}\right)=0, \\
\operatorname{Re}\left(z_{1} e^{-i \varphi}\right)=\operatorname{Re}\left(\left|z_{1}\right| e^{i(\pi+\varphi)}\right)=-\left|z_{1}\right| \cos \varphi<\lambda_{1} \cos \varphi, \\
\left|\operatorname{Re}\left(z_{2} e^{-i \varphi}\right)\right|=\left|\operatorname{Re}\left(\left|z_{2}\right| e^{i(\pi-\varphi)}\right)\right|=\left|z_{2}\right| \cos \varphi<\lambda_{2} \cos \varphi,
\end{gathered}
$$

which had to be proved.

\section{References}

[1] Antonova T.M. On convergence criteria for branched continued fraction. Carpathian Math. Publ. 2020,12 (1), 157-164. doi:10.15330/cmp.12.1.157-164

[2] Antonova T.M., Bodnar D.I. Convergence domains for branched continued fractions of the special form. In: Approximation theory and its Applications, Proc. Inst. Math. NAS Ukr., 2000, 31, 19-32. (in Ukrainian)

[3] Antonova T.M., Dmytryshyn R.I. Truncation error bounds for branched continued fraction $\sum_{i_{1}=1}^{N} \frac{a_{i(1)}}{1}+$ $\sum_{i_{2}=1}^{i_{1}} \frac{a_{i(2)}}{1}+\sum_{i_{3}=1}^{i_{2}} \frac{a_{i(3)}}{1}+\ldots$ Ukrainian Math. J. 2020, 72 (7), 1018-1029. doi:10.1007/s11253-020-01841-7 (translation of Ukrain. Mat. Zh. 2020, 72 (7), 877-885. doi:10.37863/umzh.v72i7.2342 (in Ukrainian))

[4] Antonova T.M., Dmytryshyn R.I. Truncation error bounds for branched continued fraction whose partial denominators are equal to unity. Mat. Stud. 2020, 54 (1), 3-14. doi:10.30970/ms.54.1.3-14

[5] Antonova T., Dmytryshyn R., Kravtsiv V. Branched continued fraction expansions of Horn's hypergeometric function $H 3$ ratios. Mathematics 2021, 9 (2), 148. doi:10.3390/math9020148

[6] Antonova T.M., Hoyenko N.P. Approximation of Lauricella's functions $F_{D}$ ratio by Nörlund's branched continued fraction in the complex domain. Mat. Metody Fiz.-Mekh. Polya 2004, 47 (2), 7-15. (in Ukrainian)

[7] Antonova T.M., Sus' O.M. Sufficient conditions for the equivalent convergence of sequences of different approximants for two-dimensional continued fractions. J. Math. Sci. 2018, 228 (1), 1-10. doi:10.1007/s10958-017-3601-3 (translation of Mat. Metody Fiz.-Mekh. Polya 2015, 58 (4), 7-14. (in Ukrainian))

[8] Bilanyk I.B. A truncation error bound for some branched continued fractions of the special form. Mat. Stud. $2019, \mathbf{5 2}$ (9), 115-123. doi:10.30970/ms.52.2.115-123 
[9] Bodnar D.I. Branched continued fractions. Naukova Dumka, Kiev, 1986. (in Russian)

[10] Dmytryshyn R.I. On some of convergence domains of multidimensional S-fractions with independent variables. Carpathian Math. Publ. 2019, 11 (1), 54-58. doi:10.15330/cmp.11.1.54-58

[11] Dmytryshyn R.I. Multidimensional regular C-fraction with independent variables corresponding to formal multiple power series. Proc. Roy. Soc. Edinburgh Sect. A 2020, 150 (4), 153-1870. doi:10.1017/prm.2019.2

[12] Dmytryshyn R.I. Convergence of multidimensional A- and J-fractions with independent variables. Comput. Methods Funct. Theory 2021. doi:10.1007/s40315-021-00377-6

[13] Erdélyi A., Magnus W., Oberhettinger F., Tricomi F.G. Higher transcendental functions. Vol. 1. McGraw-Hill Book Co., New York, 1953.

[14] Hoyenko N.P., Hladun V.R., Manzij O.S. On the infinite remains of the Nörlund branched continued fraction for Appell hypergeometric functions. Carpathian Math. Publ. 2014, 6 (1), 11-25. doi:10.15330/cmp.6.1.11-25 (in Ukrainian)

[15] Jones W.B., Thron W.J. Continued fractions: analytic theory and applications. Addison-Wesley Pub. Co., Reading, 1980.

[16] Lorentzen L., Waadeland H. Continued fractions with applications. Noth Holland, Amsterdam, 1992.

[17] Manzii O.S. Investigation of expansion of the ratio of Appel hypergeometric functions $F_{3}$ into a branching continued fraction. In: Approximation theory and its Applications, Proc. Inst. Math. NAS Ukr. 2000, 31, 344-353. (in Ukrainian)

Антонова Т.М. Про збіжність розвинень відномень гіпергеометричних функиій Горна Нз у гіллясті ланцюгові дроби // Карпатські матем. публ. - 2021. - Т.13, №3. - С. 642-650.

Стаття присвячена дослідженню збіжності гіллястого ланцюгового дробу з двома гілками розгалужень, який використовується для наближення вілношень гіпергеометричної функції Горна $H_{3}(a, b ; c ; \mathbf{z})$. Розглянуто випадок дійсних параметрів $c \geq a \geq 0, c \geq b \geq 0, c \neq 0$ і комплексної змінної $\mathbf{z}=\left(z_{1}, z_{2}\right)$. Спочатку доведено збіжність гіллястого ланцюгового дробу для $\mathbf{z} \in G_{\mathbf{h}}$, де $G_{\mathbf{h}}-$ двовимірний круг. Використовуючи цей результат, встановлено достатні умови рівномірної збіжності вищезгаданого гіллястого ланцюгового дробу на кожній компактній підмножині області $H=\bigcup_{\varphi \in(-\pi / 2, \pi / 2)} G_{\varphi}$, де

$$
\begin{aligned}
G_{\varphi}=\left\{\mathbf{z} \in \mathbb{C}^{2}:\right. & \operatorname{Re}\left(z_{1} e^{-i \varphi}\right)<\lambda_{1} \cos \varphi,\left|\operatorname{Re}\left(z_{2} e^{-i \varphi}\right)\right|<\lambda_{2} \cos \varphi, \\
& \left.\left|z_{k}\right|+\operatorname{Re}\left(z_{k} e^{-2 i \varphi}\right)<v_{k} \cos ^{2} \varphi, k=1,2 ;\left|z_{1} z_{2}\right|-\operatorname{Re}\left(z_{1} z_{2} e^{-2 \varphi}\right)<v_{3} \cos ^{2} \varphi\right\} .
\end{aligned}
$$

Ключові слова і фрази: гіпергеометрична функція Горна $H_{3}$, гіллястий ланщюговий дріб, збіжність. 